\title{
INFARTO AGUDO DO MIOCÁRDIO: MANUAL DE ORIENTAÇÃO PARA O PACIENTE.
}

\author{
Fernanda Carneiro Mussi* \\ Marita Guerrero** \\ Bruno Caramelli***
}

MUSSI, F.C. et al. Infarto Agudo do Miocárdio: manual de orientaçăo para o paciente. Rev.Esc.Enf.USP. v.29. n.2. p.158-65. ago. 1995

Baseado em pesquisa das expectativas de 30 pacientes internados em unidade coronária, os autores apresentam $u \mathrm{~m}$ manual de orientação para o paciente com diagnóstico de Infarto Agudo do Miocárdio. O objetivo do manual é ser um complemento do tratamento dos pacientes com Infarto do Miocárdio, a principal causa de morte em nosso país. $\dot{E}$ composto por informaçōes sobre o que é a doença, os fatores de risco a ela associados, retorno as atividades e tratamento.

INITERMOS:Doenças cardiovasculares. Infarto do Miocárdio. Educação em Saúde

\section{INTRODUÇÃO}

As doenças cardiovasculares são responsáveis por $32 \%$ das morles na cidade de São Paulo. Como doença individual. sem dúvida. o infarto do miocáadio é o maior responsável por estec alto índice lisforços têm sido realizados pelos cardiologristas do mundo todo no sentido de modificar esta situaœão. Assim. com a criação das modernas Unidades Coronarianas. com o advento do tratamento trombolítico nas primeiras horas do infarto e o uso dos beta-bloqueadores. antiagregantes plaquetários ebloqueadores da enzima de conversão do angiotensinogênio vem diminuindo essess números.

\footnotetext{
* Enfermeira. Professora Assistente da Disciplina Enfermagem Cirúrgica I e II da Faculdade de Enfermagem do Hospital Israelita Albert Einsten

* Enfermeira. Professora da Escola de Auxiliar de Enfermagem do Instituto do Coraçá do Hospital das Clínicas - FMUSP

*** Médico do Instituto do Coraçåo do Hospital das Clínicas-FMUSP.
} 
No entanto. esses benefícios vem sendo obtidos para uma minoria dos pacientes com infarto. Dados estatísticos revelam que somente cerca de 1/3 dos pacientes que morrem de infarto podem receber este tipo de tratamento? Isto se deve a vários fatores. O primeiro deles e que mais de $60 \%$ dos pacientes que morrem de infarto o fazem antes de chegar ao hospital ${ }^{3}$. () desconhecimento dos sintomas do infarto e a característica dessa doença em apresentar arritmias cardiacas fatais nas duas primeiras horas são responsáveis por esses resultados. Nlém disto, o infarto é na maioria das vezes a primeira manifestação da doença coronariana. São características fundamentais desta doença que fazem com que sua abordagem não possa se resumir ao tratamento intra-hospitalar.

Nesta situação. os profissionais de saúde interagindo com o paciente. quer na área hospitalar ou de saúde pública têm a possibilidade de discutir com o paciente/cliente sobre os fatores de risco para doença isquêmica do coração aos quais uma pessoa se expõe e a forma de tentar previnir os agravos a saúde. Segundo (YHII) $\Lambda^{4}$ é importante ajuda-lo a reconhecer os direitos a saúde e os recursos que deverão estar ao seu alcance para a manutenção de sua saúde.

No período em que o paciente com infarto do miocárdio permanece hospitalizado o enfermeiro tem um papel preponderante nas atividades educativas. dando oportunidade ao indivíduo de conhecer e acompanhar o curso da doença e tratamento. compreender as medidas preventivas frente a evolução da doença e a julgar o que o melhor para ele sempre que possivel. maximizando assim as possibilidades de obter sua recuperação. Ressalta-se que. na Classificação Brasileira de (cupaçoes² e na leei que dispõe sobre a Regulamentação do Exercício da Enfermagem ${ }^{1}$. constituem-se como função básica do enfermeiro orientar sobre cuidados de enfermagem e informar em matéria de saúde.

Limbora a assimilação de novos concejtos a a mudança de comportamento não dependam apenas de informação em materia de saúde por excelente que seja. acreditamos que conhecer solbe a doenca of um pré-requisito para que a aderência ao tratamento seja possível.

Partindo da premissa de que as atividades educativas sistematizadas e lundamentadas nas características e necessidades dos pacientes são consideradas de grande importancia foi elaborado. anteriormente ${ }^{6}$. um estudo com a finalidade de identificar as expectativas de orientação de 30 pacientes com Insulieiencia (oronariana $\Lambda$ guda. Nessa pesquisa ficou evidenciada a predominância de expectativas relativas a atividade física, prevenção da doença. tratamento. atividade sexual. exames e alimentação. Quanto a natureza das expectativas a maioria dos pacientes mencionou sobre: o que podem ou não fazer: possibilidade de retornar ao babalho: como evitar a doença: fatores de risco: evolução e causa da doença: finalidade do tratamento: possibilidades 
de retorno a atividade sexual: resultados dos exames e alimentos e líquidos permitidos ${ }^{6}$. Baseado nesse estudo elaborou-se un Manual de Orientação para o Paciente com Infarto Agudo do Miocárdio.

Dessa forma. objetiva-se no presente trabalho apresentar o conteúdo deste manual como contribuição para atender as necessidades de orientaçóes dos pacientes com Infarto Agudo do Miocárdio e conseqüentemente? minimizar as suas tensōes e dúvidas quanto ao seguimento do tratamento o perspectivas de qualidade de vida.

Enfatizamos que a implementação de um Manual de (Orientaşa nem sempre esgota. no conteúdo proposto as necessidades daquele para quem se destina cabendo aos profissionais envolvidos com os indivíducis detecta-las e atendê-las na medida do possível.

\section{APRESENTAÇÃo DO CONTEÚdo DO MANUAL DE ORIENTAÇÃO DO PACIENTE COM INFARTO AGUDO DO MIOCÁRDIO.}

\section{MANUAL DE ORIENTAÇÃO DO PACIENTE COM INFARTO AGUDO DO MIOCÁRDIO}

Este manual pretende ser un complemento ao tratamento dos pacientes com Infarto Agudo do Miocárdio. De lorma alguma as informacões aqui contidas devem sulstiluir as orientacones do médico. enfermeiro. nutricionista. psicólogo. assistente social efisioterapeuta mas complementálas. para molhor compreensão do Infarto $\Lambda$ gudo do Miocárdio.

E importanle lembrar que muitas pesseras que tiveram infarto podem o devem voltar à vida normal. $\Lambda$ equipe de saúde pretende. com os recursos de que dispõe. ajudá-lo no retorno as atividades. mas a sua contribuição 6 importante para alcançarmos juntos os oljetivos de seu tratamento e recuperaç̃ò.

\section{- O QUE É O INFARTO AGUDO DO MIOCÁRDIO?}

() miocárdio (músculo que forma o coração) recobe oxigênio levado pelo sangue atraves de vasos chamados de arterias coronarianas. $\Lambda$ doenca res. ponsável pela obstrução (entupimento) destas artérias. chama-se atcorosclerose coronariana. 
É provocada pela deposição de "gorduras" nas paredes das artérias. impedindo que o sangue rico em oxigênio chegue ao miocárdio.

Quando há um "entupimento" nas artérias por um período prolongado ocorre o Infarto Agudo do Miocárdio. $\Lambda$ parte do coração que para de receber sangue perde a sua função sendo. mais tarde. substituída por uma "cicatriz".

\section{- FATORES DE RISCO:}

Sabemos que certas condiçoes chamadas de F $\Lambda$ TORES I)E RIS(C) contriluem para que voce desenvolva a aterosclerose coronariana e como conseqüencia o infarto. Vale lembrar que a combinaçáa de dois ou mais fatores aumenta esta probabilidade. Portanto o grande segredo para evitar a progressão da doença e o risco de um novo infarto está no controle dos fatores de risco.

São eles:

\section{IIIPERTENSTOA ARTERIAL SISTEMMICA (Pressĩo All(t)}

$\Lambda$ pressão arterial e o resultado da forsa que o coraça faz para enviar o sangue para todo o corpo e que pode ser medida nas arterias do braço por meio de um aparelho. lim algumas pessoas a pressão te permanentemente alta. $\Lambda$ isto se ehama de llipertensão $\Lambda$ rterial. $\Lambda$ hipertensão arterial pode acelerar a aterosclerose coronariana responsável pela obstrucão das arterias coronarianas. () controle da hipertensão é necessário para prevenir complicaçōes como o Infarto do Miocárdio. Insuficiência Renal e Derrame (erebral.

\section{TABICISMO}

() riseo de infarto (e sempre maior nos fumantes. A nicolina do cigarro pode provocar uma vasoconstrição (estreitamento) da artoria capaz de reduzir a olerta de sangue pelas artérias coronarianas. Alem disso. a nicotina pode aumentar a pressão arterial e a freqüencia cardíaca (número de batimentos do coração por minuto). () dano do fumo (e tanto maior quanto maior a quantidade de eigarros fumados por dia e a duracão do vício. () riseo não diminui com cigarros de baixos teores.

\section{SEIDENT:IRISMO}

$\Lambda$ lguns estudes mostram que pessoas que não fazem exercício regularmente correm maior riseo de infarto. () excrécio tamberm ajuda a evitar o excesso de pesso. 


\section{DI: BBETES}

() diabete ó uma doença na qual o corpo não produz uma quantidade suficiente do hormônio insulina e por isso há um excesso de açuear no sangue. () diabetes está associado com a aterosclerose eo maior riseo de infarto.

\section{ESTRESSE}

$\Lambda$ tensão emocional. o nervosismo. a vida agilada o as pressões a que somos submetides diariamente no trabalto e em casa fazen parte do que o conhecido como estresse.

Estudos mostraram que individuos submetides a cestresse constante. apresentam maior riseo de infarto.

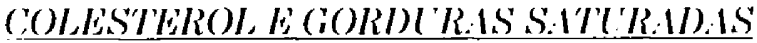

() colesterol o uma grordura fabricada em nosso corpo a que também está presente nos alimentes que comenos.

() excesso podera depositar-so nas arterias o contribuir para a ateroselerose. As gorduras insalturadas, que sion encontradas em peixes, aves e óleos vegetais devem ser preferoncialmento consumidas pois produzem menos colesterol.

\section{- DIETA}

Mantenha seu corposoly controle. () exeesso de peso aumentad a chancer de hipertensão arterial. diabetes o niveis altos de colesterol. So você precisa

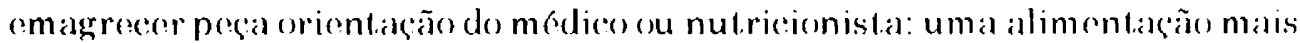
saudavel o muito importante o ajudará a protegerer seu coragãos.

Alguns cuidades são fundamentais:

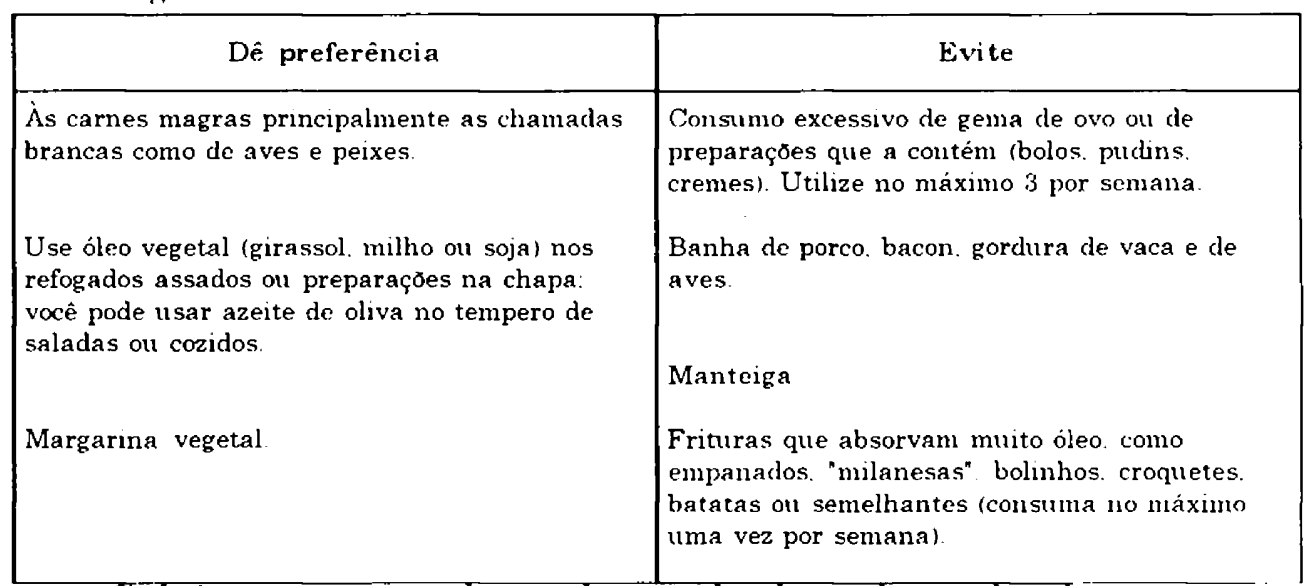

Sä: Procure usar sal e produtos salgados industrializalos (enlatados.emlsulides) eom moderaceño. 


\section{- COMO INICIAR ATIVIDADE FÍSICA APÓS O INFARTO?}

() retorno lento e gradual as atividades físicas é muito importante para a sua completa recuperação. O exercício físico diminui o risco de um novo infarto. No entanto, toda atividade física deve seguir orientação médica.

Você pode e deve desempenhar atividades diárias habituais como: tomar banho (não há necessidade de acompanhante); barbear-se, escovar os dentes. tomar e controlar suas medicações, dentre outras. liberte-se da cama e do pijama, pois a fase de maiores restriçoes foi superada durante a hospitalização.

Caminhar é um excelente exercício que deve ser realizado diariamente. Faça pequenas cam inhadas aumentando o percurso gradativamente. Ande em lugar plano, evite escadas e durante as primeiras caminhadas fora de casa esteja acompanhado.

Mais tarde, após a liberação médica você poderá aumentar o percurso de suas caminhadas e, até mesmo, praticar esportes ou dirigir automóveis.

livite carregar ou levantar pesos. malas ou crianças, pois esse tipo de excrécio acarreta uma sobrecarga para o coração.

Retorne as suas atividades sociais, podendo receber visitas. participar de pequenas reuniōes que não lhe causem estresse. No princípio evite gran. des festas e reuniões longas, principalmente entre fumantes. Atividades de lazer são importantes. Você poderá executar atividades leves como assistir 'TV. ouvir música. ler. escrever. fazer tricô ou crochê, dentre outras.

\section{- ATIVIDADE SEXUAL: QUANDO E COMO INICIAR?}

$\Lambda$ atividade sexual poderá ser reiniciada aproximadamente 30 dias após o infarto. () retorno deve ocorrer de forma gradual utilizando posições cômodas e evitando atividade sexual após alimentação ou ingestão de bebidas al. coolicas.

Nesta fase, de retorno as atividades física, social e sexual, e importante que você esteja atento a sensaçoes como: cansaço, falta de ar. palpitaçoes. opressão ou dor no peito. sudorese. e comunique o seu médico se elas ocorrerem.

\section{QUANDO RETORNAR AO TRABALHO?}

$\Lambda$ volta ao trabalho (e um dos objetivos da reabilitação cardiológica. () retorno ao trabalho está previsto para 2 meses após a infarto sempre que possível. 


\section{PODEREI VIAJAR?}

Nos primeiros dois meses após o infarto não b aconselhável que você viaje. Depois disso, comece com lugares proximos evitando as viagens longas e cansativas. Nas primeiras vezes, se você for de carro, outra pessoa deverá dirigir.

\section{- TRATAMENTO}

$\Lambda$ pos o infarto, alguns pacientes poderão ser operades. ou seja, submetidos a cirurgia de ponte de safena, chamada de Revascularização do Miocárdio. Porém a maioria poderá ser tratada com medicamentos e pelo controle dos fatores de risco.

() cateterismo o um exame que permite identificar o grau e a localizacão da obstrução (entupimento) das artérias coronarianas. liste exame pode ajudar a definir o tipo de tratamento ou até mesmo "desentupir" a artéria coronariana por mejo de uma técenica chamada de Angioplastia.

() acompanhamento médico periódieo é importante para diagnosticar precocemente a progressão da doença e para a tomada de providencias antess que alguma conseqüencia grave ocorra.

Esperamos que algumas de suas dúvidas tenham sido esclarecidas com este manual.

Não esqueça! Se voltarem os sintomas do infarto procure imediatament.e o seu médico ou o hospital.

MUSSI. F.C. et al. Acute myocardial infarction: content of a manual Rev. Esc. Enf.USP. v.29. n.2.p.158.65. aug. 1995

The authors present a booklet for the patients with acute myocardial infarction. The main purpose of the booklet is to be a complement in the treatment for myocardial infarction patients, the leading cause of death in Brazil. It contains information about the disease, risk factors, return to work and treatment.

INITERMS:Cardiovascular diseases. Myocardial enfarction. Health education. 


\section{REFERÊNCIAS BIBLIOGRÁFICAS}

1. BRASIL. Leis etc. Lei n. 7.498 de 25 de junho de 1986. Dispó sobre a regulamentação do exercício da enfermagem e da outras providencias. Diário Oficial da União. Brasília. 26 jun. 1986. Seção I, p.9273-5.

2. BRASIL. Ministério do trabalho. Secretaria de Emprego e Salário. Classificação Brasileira de Ocupaçes. CBO. Rio de Janeiro. 1975.

3. BRAUNWALD, E. Acute myocardial infartcion. In: PASTERNACK, R. C.: BRAUNWALD. E.: SOBEL, B. E. Heart disease: textbook of cardiovascular medicine. 4 ed. Philadelphia. W. B. Saunders Company. 1992. cap. 39. p. 120-9.

4. CHIDA, A. M. Orientaçâo sistematizada a pacientes internados com doença transmissível. Sáo Paulo. 1990. 151 p. Tese (Doutorado)-Escola de Enfermagem. Universidade de São Paulo.

5. LOTUFO. P. A.: LOLIO, C. A. D. Tendência da mortalidade por doença isquêmica do coraçăo no estado de Såo Paulo. 1970-1989. Rev. Arq. Bras. Cardiol. v. 61. n. 3. p. 149-53. 1993.

6. MUSSI, F. C.: GUERRERO, M. Expectativas do paciente coronariano hospitalizado: proposta de um programa de orientaçà Rev. Soc. Cardiol. Est. SP . n.4. p.15-22. 1990.

7. TOPOL. E. J. Textbook of intervention cardiology. Philadelphia. W. B. Saunders Company. 1990. cap. 5. p. 76-120: Thrombolytic Intervention 